\title{
openheart Cardiovascular disease risk factors in relation to smoking behaviour and history: a population-based cohort study
}

\author{
Jaana Keto, ${ }^{1,2}$ Hanna Ventola, ${ }^{2,3}$ Jari Jokelainen, ${ }^{1,4}$ Kari Linden, ${ }^{5}$ \\ Sirkka Keinänen-Kiukaanniemi, ${ }^{1}$ Markku Timonen, ${ }^{1}$ Tero Ylisaukko-oja, ${ }^{1,2}$ \\ Juha Auvinen ${ }^{1}$
}

To cite: Keto J, Ventola $\mathrm{H}$ Jokelainen $\mathrm{J}$, et al. Cardiovascular disease risk factors in relation to smoking behaviour and history: a population-based cohort study. Open Heart 2016;3: e000358. doi:10.1136/ openhrt-2015-000358

Received 1 November 2015 Revised 1 June 2016 Accepted 7 June 2016

\section{(a) CrossMark}

${ }^{1}$ Faculty of Medicine, Center for Life Course Health Research, University of Oulu, Oulu, Finland

${ }^{2}$ MedEngine Oy, Helsinki, Finland

${ }^{3}$ Department of

Pharmacology, Institute of Biomedicine, University of Helsinki, Helsinki, Finland ${ }^{4}$ Unit of General Practice, Oulu University Hospital, Oulu, Finland

${ }^{5}$ Pfizer Oy, Helsinki, Finland

Correspondence to Jaana Keto;

jaana.keto@helsinki.fi

\section{ABSTRACT}

Objective: To investigate how individual risk factors for cardiovascular disease (CVD) (blood pressure, lipid levels, body mass index, waist and hip circumference, use of antihypertensive or hypolipidemic medication, and diagnosed diabetes) differ in people aged 46 years with different smoking behaviour and history.

Methods: This population-based cohort study is based on longitudinal data from the Northern Finland Birth Cohort 1966 project. Data were collected at the 31-year and 46-year follow-ups, when a total of 5038 and 5974 individuals participated in clinical examinations and questionnaires. Data from both follow-ups were available for 3548 participants. In addition to individual CVD risk factors, Framingham and Systematic Coronary Risk Evaluation (SCORE) algorithms were used to assess the absolute risk of a CVD event within the next decade.

Results: The differences in individual risk factors for CVD reached statistical significance for some groups, but the differences were not consistent or clinically significant. There were no clinically significant differences in CVD risk as measured by Framingham or SCORE algorithms between never smokers, recent quitters and former smokers $(7.5 \%, 7.4 \%, 8.1 \%$ for men; $3.3 \%, 3.0 \%, 3.2 \%$ for women; $p<0.001)$.

Conclusions: The effect of past or present smoking on individual CVD risk parameters such as blood pressure and cholesterol seems to be of clinically minor significance in people aged 46 years. In other words, smoking seems to be above all an independent risk factor for CVD in the working-age population. Quitting smoking in working age may thus reduce calculated CVD risk nearly to the same level with people who have never smoked.

\section{INTRODUCTION}

Smoking is the most important preventable cause of death in the Western world, causing some 5 million premature deaths globally each year. ${ }^{1}$ Cigarette smoke is a major risk factor for cardiovascular disease (CVD) and the second leading cause for CVD mortality after high blood pressure. Other major risk

\section{KEY QUESTIONS}

What is already known about this subject?

- Smoking significantly raises the risk of cardiovascular disease (CVD). The difference in CVD risk between smokers and non-smokers becomes significant in middle age, especially for men. The time it takes for the CVD risk of a former smoker to reach the same level as that of a never smoker has been estimated to be as long as 15 years.

What does this study add?

- The effect of past or present smoking on several individual CVD risk parameters such as blood pressure and cholesterol seems to be of clinically minor significance in people aged 46 years. Quitting smoking in working age might thus reduce calculated CVD risk to the same level with people who have never smoked.

How might this impact on clinical practice?

- We have shown that smoking history does not seem to have a strong or slowly reversible effect on cardiovascular risk factors at the age of 46 . In other words, successful smoking cessation taking place as late as in working age has great preventative cardiovascular outcomes. This result may encourage physicians to support their patients more in smoking cessation.

factors for CVD mortality include high blood glucose, physical inactivity, obesity and elevated cholesterol levels, all of which are associated with smoking according to previous research. ${ }^{2}$ CVD accounts for $>17$ million deaths globally each year, of which more than $10 \%$ are estimated to be caused by cigarette smoke. ${ }^{23}$

According to the WHO, more than 1 billion people smoke, and the prevalence is constantly rising. ${ }^{4}$ Several environmental and genetic factors contribute to the initiation of smoking, and although most smokers want to quit, they commonly continue due to strong 
nicotine addiction. ${ }^{5}$ Smoking is more common among men and in lower socioeconomic classes, appearing concomitantly with other harmful health behaviour. ${ }^{6}$ Men also have a two to five times higher risk of CVD. ${ }^{7}$ These factors contribute to the substantial gender imbalance in life expectancy. In Finland, for example, life expectancy is 77.5 years for men and 83.4 years for women. ${ }^{8}$

In this study, we set out to investigate gender-specific CVD risk factors in relation to smoking status and history in the population aged 46 years. We employed the well-established Framingham risk score as a tool to estimate risk of a CVD event within the next 10 years among current smokers, individuals who had quit smoking recently or in the past and those who had never smoked. Smoking greatly raises CVD risk points in the Framingham equation, but other risk parameters, such as blood pressure, are included as well. We hypothesised that past smoking would be visible as an increase in these risk parameters and thus raise the risk of a CVD event for a former smoker when compared with a never smoker. In other words, we expected to find significant differences in CVD risk factors between recent quitters, former smokers and never smokers. We also hypothesised that current smokers would have significantly higher rates of individual CVD risk factors than non-smokers.

The analyses were based on the unique, prospective, population-based Northern Finland Birth Cohort 1966 study sample, which consists of $>5000$ well-characterised individuals. As serious cardiovascular events often take place in the population aged 60 and over, it is of key importance to characterise the risk of a CVD event within the next decade for people in their late 40s.

\section{METHODS}

\section{Study population}

This study is based on the Northern Finland Birth Cohort 1966 study sample, which is a longitudinal research programme in Finland's two northernmost provinces. The study is based on clinical measurements and questionnaires performed at examinations during a 31-year follow-up in 1997 and during a 46-year follow-up in 2012. All participants provided written informed consent, and the Ethical Committee of the Northern Ostrobothnia Hospital District has approved the Northern Finland Birth Cohort study (\$94/2011), which is performed according to the Declaration of Helsinki of 1983.

The Northern Finland Birth Cohort 1966 initially included all of the 12058 children whose expected time of delivery was in 1966. The entire cohort has been followed up since pregnancy with clinical examinations and questionnaires covering health, lifestyle and socioeconomic indicators at $0,1,14,31$ and 46 years of age. The present study is based on the 31-year and 46-year follow-ups, in which 5038 and 5974 individuals participated, respectively. The CVD risk estimation carried out in this study was based on smoking status and other risk parameters mapped at the age of 46. The 31-year smoking status was used to investigate changes in smoking habits and development of CVD risk factors between ages 31 and 46. This analysis could be performed on 3548 participants for whom there were data from both follow-ups. Study sample selection is presented in figure 1. Characteristics of the study sample are presented in table 1 .

\section{Clinical examinations}

During the 31-year and 46-year follow-up examinations, the participants gave blood samples and underwent physical examination, anthropometry and blood pressure determination performed by a study nurse or physician.

Weight and height, as well as waist and hip circumference, were measured with the participants wearing light underwear. Body height and weight were measured to an accuracy of $0.1 \mathrm{~cm}$ and $0.1 \mathrm{~kg}$, respectively. Based on these measurements, body mass index (BMI; $\mathrm{kg} / \mathrm{m}^{2}$ ) was calculated. The waist-to-hip ratio (WHR) was also calculated.

Systolic and diastolic blood pressure was measured on the right arm of the seated participants using an automated oscillometric blood pressure device (Omron

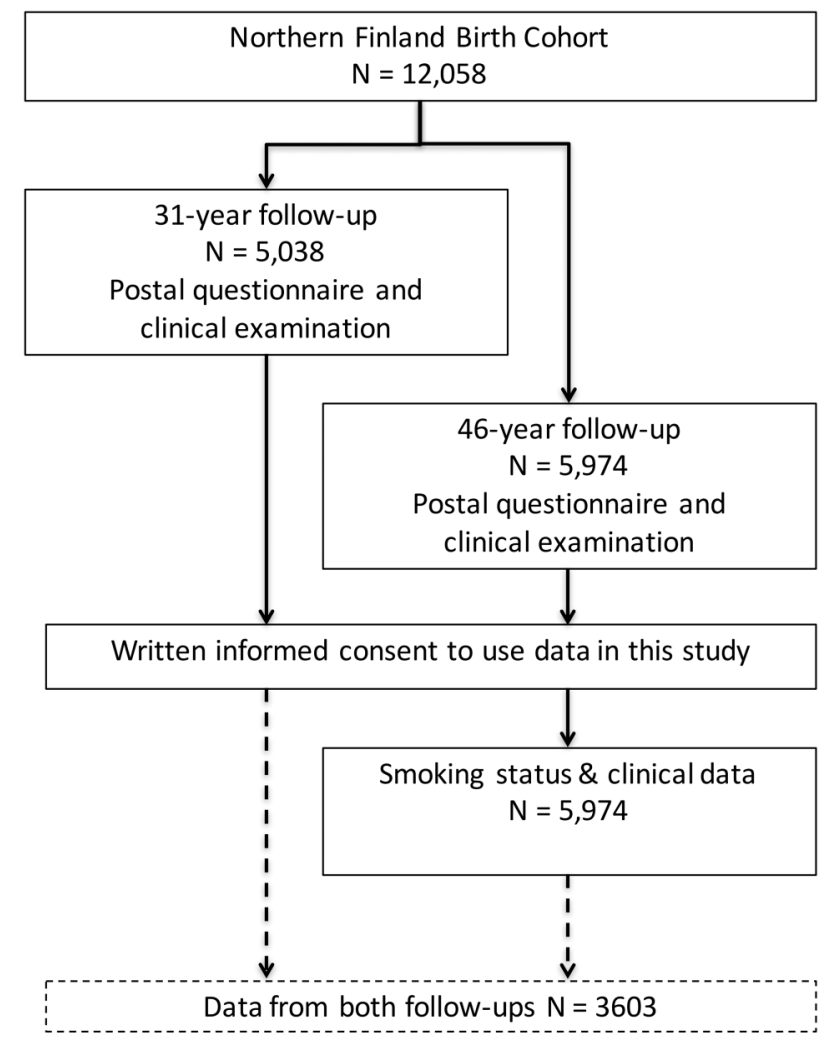

Figure 1 Selection of the study sample. Data from the 46-year follow-up were used to assess the relationship between smoking history and cardiovascular risk factors. Data from the 31-year and 46-year follow-ups were used to assess changes in smoking status, that is, determine the smoking history. 
Table 1 Characteristics of the study population at the age of 46

\begin{tabular}{|c|c|c|}
\hline Database & $\begin{array}{l}\text { Men } \\
\text { NFBC } 1966\end{array}$ & $\begin{array}{l}\text { Women } \\
\text { NFBC } 1966\end{array}$ \\
\hline $\mathrm{n}$ & 2736 & 3238 \\
\hline Age (years) & $46.6 \pm 0.6$ & $46.6 \pm 0.6$ \\
\hline Current smokers, n (\%) & $743(27.5)$ & $665(20.3)$ \\
\hline Recent quitters, n (\%) & $64(2.3)$ & $57(1.8)$ \\
\hline Former smokers n, (\%) & $707(25.8)$ & $694(21.4)$ \\
\hline Never smokers $n,(\%)$ & $1222(44.7)$ & $1822(56)$ \\
\hline Use of antihypertensive medication (\%) & 18.5 & 16.6 \\
\hline Use of hypolipidemic medication (\%) & 6.9 & 2.3 \\
\hline Type 2 diabetes (\%) & 3.0 & 2.6 \\
\hline $\mathrm{BMI}$ & $27.3 \pm 4.3$ & $26.5 \pm 5.2$ \\
\hline Waist (cm) & $97.5 \pm 11.8$ & $87.3 \pm 13.1$ \\
\hline Hip $(\mathrm{cm})$ & $99.3 \pm 7.8$ & $100.5 \pm 10.9$ \\
\hline WHR & $0.98 \pm 0.06$ & $0.87 \pm 0.06$ \\
\hline Total cholesterol (mmol/L) & $5.54 \pm 1.0$ & $5.18 \pm 0.85$ \\
\hline $\mathrm{HDL}(\mathrm{mmol} / \mathrm{L})$ & $1.40 \pm 0.33$ & $1.67 \pm 0.39$ \\
\hline LDL (mmol/L) & $3.74 \pm 0.94$ & $3.24 \pm 0.86$ \\
\hline Triglycerides (mmol/L) & $1.49 \pm 0.96$ & $1.08 \pm 0.58$ \\
\hline Systolic blood pressure $(\mathrm{mm} \mathrm{Hg})$ & $128.2 \pm 13.9$ & $118.51 \pm 15.5$ \\
\hline Diastolic blood pressure (mm Hg) & $86.5 \pm 10.2$ & $82.4 \pm 10.7$ \\
\hline SCORE risk score (\%) & $1.7 \pm 0.8$ & $0.3 \pm 0.2$ \\
\hline Framingham risk score (\%) & $9.4 \pm 5.1$ & $3.7 \pm 2.6$ \\
\hline
\end{tabular}

The values are presented as mean \pm SD unless otherwise indicated.

BMI, body mass index; HDL, high-density lipoprotein; LDL, low-density lipoprotein; NFBC 1966, Northern Finland Birth Cohort 1966; SCORE, Systematic Coronary Risk Evaluation; WHR, waist to hip ratio.

Digital Automatic Blood Pressure Monitor Model M10-IT) and an appropriately sized cuff. The mean of two lowest values out of three measurements was recorded as blood pressure.

Serum total cholesterol, high-density lipoprotein (HDL) and low-density lipoprotein (LDL) cholesterol, and triglycerides were determined from fasting samples using an enzymatic assay method. All plasma samples were analysed in a University Hospital of Oulu laboratory according to a standardised protocol.

Information on diagnosed type 2 diabetes, in addition to the use of antihypertensive and hypolipidemic medication, was acquired by a questionnaire at the 31-year and 46-year follow-ups.

\section{Smoking status and study groups}

Smoking status assessment was based on standards of the Finland Cardiovascular Risk (FINRISK) study and four smoking-related questions ('Have you ever smoked?', 'Have you ever smoked regularly, almost daily for at least a year?', 'Do you smoke now?' and 'When was the last time you smoked?') collected at the 31-year and 46-year follow-ups. ${ }^{9}$ Participants were divided into four groups based on their smoking status at age 46: (1) current smokers ( $n=1408$; 665 women, 743 men), (2) recent quitters ( $\mathrm{n}=121 ; 57$ women, 64 men), (3) former smokers $(n=1401 ; 694$ women, 707 men) and (4) never smokers ( $\mathrm{n}=3044 ; 1822$ women, 1222 men). Those who answered ' $n o$ ' to a question 'Have you ever smoked?' were considered never smokers. Those who had smoked almost daily for at least a year but had quit at least 6 months ago were considered former smokers, whereas those who had quit more recently were considered recent quitters. Those who smoked 2-7 days a week or whose last smoke was today or yesterday were considered current smokers.

\section{CVD risk factor assessment}

\section{Framingham risk score}

We adopted the gender-specific 2008 Framingham risk score prediction equation by D'Agostino et al ${ }^{10}$ to estimate the individual's absolute risk (\%) for a first cardiovascular event within 10 years. The events assessed were fatal or non-fatal coronary heart disease, stroke, peripheral artery disease or heart failure. Parameters included in the algorithm are smoking status, age, systolic blood pressure, total cholesterol, HDL cholesterol, type 2 diabetes status and treatment for hypertension. All parameters have varying contribution to the total score, age being the variable with main effect. The method is based on a cohort of inhabitants of Framingham, Massachusetts, with the mean age of 49. The method has since been validated for the white, middle-aged population, and can be thought to estimate cardiovascular risk in our population fairly well without further ethnical calibration. ${ }^{10}$

Systematic Coronary Risk Evaluation (SCORE)

The Systematic Coronary Risk Evaluation (SCORE) algorithm includes smoking status, age, systolic blood 
pressure and total cholesterol as risk factors for a fatal cardiovascular event in the next 10 years. ${ }^{11}$ The SCORE risk was calculated for each participant according to a gender-specific algorithm. The high-risk population version of the SCORE algorithm we used is based on Finnish, Danish and Norwegian cohorts, and is thus especially well applicable for our data set. Including the SCORE algorithm in our study was thus also a means to validate the Framingham method for our population, even though the end points are slightly different for these two models.

\section{Other CVD risk factors}

In addition to the risk factors included in the Framingham and SCORE equations, we examined weight, BMI, waist and hip circumference, serum triglycerides and LDL cholesterol, which are known risk factors for CVD.

\section{Statistical analysis}

Distributions of continuous variables were expressed as means $\pm \mathrm{SD}$, and categorical variables as numbers and percentage of proportions. Log transformation was used to normalise the skewness of the distributions. The $\chi^{2}$ test, independent-sample t-test or analysis of variance (ANOVA) were used to study the association between different smoking habits and cardiovascular risk factors. Logistic regression analysis was used to examine the associations between smoking habits and binary cardiovascular risk factors. Moreover, analysis of covariance (ANCOVA) was used to examine the association between smoking habits and known cardiovascular risk factors. Post hoc analyses between the groups were performed using multiplecomparison Dunnett's test, and current smokers were used as control group. Potential confounding factors, BMI and the use of antihypertensive medication or hypolipidemic medication, as appropriate, were controlled in multivariate analysis. A $\mathrm{p}<0.05$ was considered statistically significant. Statistical analyses were conducted using the open-source software package R V.3.1.0.

\section{RESULTS}

Of the 12058 live-born participants, 11322 participants were alive and their addresses were known at the age of 31 and 10282 participants at the age of 46. At the 31-year time point, 5038 people answered the questionnaire and participated in clinical examinations. In the 46-year follow-up, the corresponding number was 5974. Data from the 31-year and 46-year follow-ups were available for 3548 participants, who had given written informed consent to use their data in this study (figure 1).

\section{Smoking history and individual cardiovascular risk factors}

The mean values of CVD risk factors analysed in the present study are presented in table 2. BMI was adjusted for when testing for differences between groups with different smoking histories.
$\mathrm{BMI}$, waist circumference and WHR

Smokers had a higher BMI and greater waist circumference than individuals who had never smoked $(\mathrm{p}<0.001$ for both genders).

Cholesterol, triglycerides and use of hypolipidemic medication

Currently smoking men had higher total cholesterol $(p<0.028)$ and triglyceride levels $(p<0.001)$ than never smokers. Currently smoking women also had a significantly poorer lipid profile than never smokers: the levels of LDL and triglycerides were higher $(p=0.002$ and $\mathrm{p}<0.001$, respectively), whereas HDL was lower $(\mathrm{p}<0.001$; use of hypolipidemic medication adjusted).

Among women, currently smoking and recently quit individuals used hypolipidemic medication more often than former and never smokers $(p=0.007)$. Such a difference was not observed among men.

\section{Blood pressure}

In men, former smokers had the highest mean systolic and diastolic blood pressure $(\mathrm{p}<0.001$ and $\mathrm{p}<0.011$; use of antihypertensive medication adjusted) and they also used more antihypertensive medication than other groups $(p=0.015)$. In women, never smokers had the highest systolic blood pressure $(\mathrm{p}=0.016$; use of antihypertensive medication adjusted). No difference was found in diastolic blood pressure between the female groups.

\section{Diabetes}

Type 2 diabetes rate was similar among former and current male smokers, whereas never smokers had significantly less type 2 diabetes $(\mathrm{p}<0.001)$. For women, no statistical difference was found between the groups.

\section{Smoking history and risk of a cardiovascular event}

Framingham risk score was calculated to assess the absolute risk of a first CVD event in the next 10 years across study groups. The mean Framingham risk score for currently smoking men was $14.7 \%$, whereas the risk for never smokers was $7.5 \% \quad(p<0.001)$. For currently smoking women, the mean Framingham risk score was $5.9 \%$ and $3.3 \%$ for never smokers $(p<0.001)$. Current smokers also had a higher CVD risk in comparison with former smokers and recent quitters $(p<0.001$ for both genders). There were no clinically significant differences in the Framingham risk scores among never smokers, recent quitters and former smokers (table 2, figure 2). Smoking was also associated with a higher risk of a fatal cardiovascular event defined by the SCORE equation for both genders $(p<0.001)$, validating the Framingham risk score for CVD events (table 2).

\section{Changes in smoking behaviour over time}

At age $31,38 \%$ of men and $26 \%$ of women smoked. Approximately $40 \%$ ( $41 \%$ of men; $39 \%$ of women) had quit smoking by age 46 and $\sim 60 \%$ (60\% of men; $61 \%$ of 
Table 2 CVD risk factors in the Northern Finland Birth Cohort 1966 study sample, measured at the age of 46

\begin{tabular}{|c|c|c|c|c|c|c|c|c|c|c|}
\hline & \multicolumn{5}{|l|}{ Men } & \multicolumn{5}{|l|}{ Women } \\
\hline & $\begin{array}{l}\text { Current } \\
\text { smokers }\end{array}$ & $\begin{array}{l}\text { Recent } \\
\text { quitters }\end{array}$ & $\begin{array}{l}\text { Former } \\
\text { smokers }\end{array}$ & $\begin{array}{l}\text { Never } \\
\text { smokers }\end{array}$ & p Value & $\begin{array}{l}\text { Current } \\
\text { smokers }\end{array}$ & $\begin{array}{l}\text { Recent } \\
\text { quitters }\end{array}$ & $\begin{array}{l}\text { Former } \\
\text { smokers }\end{array}$ & $\begin{array}{l}\text { Never } \\
\text { smokers }\end{array}$ & p Value \\
\hline $\mathrm{n}$ & 743 & 64 & 707 & 1222 & & 665 & 57 & 694 & 1822 & \\
\hline BMI $\left(\mathrm{kg} / \mathrm{m}^{2}\right)$ & $27.2 \pm 4.5$ & $27.5 \pm 4.0$ & $27.9 \pm 4.0$ & $26.8 \pm 4.0$ & $<0.001^{*}$ & $27.1 \pm 5.4$ & $26.8 \pm 5.7$ & $26.9 \pm 5.4$ & $26.1 \pm 5.1$ & $<0.001^{\star}$ \\
\hline Waist $(\mathrm{cm})$ & $98.3 \pm 12.3$ & $99.6 \pm 10.9$ & $99.0 \pm 11.6$ & $95.8 \pm 11.3$ & $<0.001^{\star}$ & $89.2 \pm 13.6$ & $89.0 \pm 15.0$ & $88.5 \pm 13.5$ & $85.9 \pm 12.4$ & $<0.001^{*}$ \\
\hline Hip (cm) & $98.8 \pm 8.2$ & $99.9 \pm 8.3$ & $100.4 \pm 7.8$ & $98.9 \pm 7.3$ & $<0.001^{*}$ & $101.2 \pm 11.2$ & $101.2 \pm 12.5$ & $101.7 \pm 10.9$ & $99.7 \pm 10.8$ & $<0.001^{*}$ \\
\hline WHR & $1.0 \pm 0.1$ & $1.0 \pm 0.1$ & $1.0 \pm 0.1$ & $1.0 \pm 0.1$ & $<0.001^{\star}$ & $0.9 \pm 0.1$ & $0.9 \pm 0.1$ & $0.9 \pm 0.1$ & $0.9 \pm 0.1$ & $<0.001^{*}$ \\
\hline Total cholesterol†‡ (mmol/L) & $5.6 \pm 1.0$ & $5.5 \pm 1.0$ & $5.6 \pm 1.0$ & $5.5 \pm 1.0$ & $0.028^{\star} \S$ & $5.2 \pm 0.9$ & $5.1 \pm 0.7$ & $5.1 \pm 0.8$ & $5.2 \pm 0.8$ & $0.521^{\star} \S$ \\
\hline HDL cholesterol† (mmol/L) & $1.35 \pm 0.3$ & $1.36 \pm 0.3$ & $1.41 \pm 0.3$ & $1.42 \pm 0.3$ & $<0.001 * \S$ & $1.58 \pm 0.4$ & $1.77 \pm 0.4$ & $1.66 \pm 0.4$ & $1.69 \pm 0.4$ & $<0.001^{\star} \S$ \\
\hline LDL cholesterol (mmol/L) & $3.81 \pm 1.0$ & $3.69 \pm 0.9$ & $3.80 \pm 0.9$ & $3.66 \pm 0.9$ & $0.002 * \S$ & $3.34 \pm 1.0$ & $2.98 \pm 0.9$ & $3.21 \pm 0.8$ & $3.22 \pm 0.8$ & $0.002 * \S$ \\
\hline Triglycerides (mmol/L) & $1.7 \pm 1.0$ & $1.4 \pm 0.6$ & $1.5 \pm 1.0$ & $1.4 \pm 0.9$ & $<0.001 * \S$ & $1.2 \pm 0.6$ & $1.1 \pm 0.6$ & $1.1 \pm 0.6$ & $1.0 \pm 0.5$ & $<0.001^{\star} \S$ \\
\hline Systolic BP†‡ (mm Hg) & $127.1 \pm 13.8$ & $126.4 \pm 13.0$ & $129.5 \pm 13.4$ & $128.1 \pm 14.2$ & $0.011^{\star} \S$ & $117.5 \pm 15.4$ & $116.8 \pm 16.2$ & $117.5 \pm 15.4$ & $119.1 \pm 15.2$ & $0.016^{\star} \S$ \\
\hline Diastolic BP $(\mathrm{mm} \mathrm{Hg})$ & $86.3 \pm 10.2$ & $86.1 \pm 9.5$ & $87.8 \pm 9.8$ & $85.9 \pm 10.3$ & $<0.001 * \S$ & $82.6 \pm 11.1$ & $81.2 \pm 11.8$ & $81.6 \pm 10.7$ & $82.6 \pm 10.5$ & $0.110^{\star} \S$ \\
\hline $\begin{array}{l}\text { Antihypertensive medication } \\
(\%) \dagger\end{array}$ & 17.4 & 16.9 & 22.6 & 17.1 & $0.015^{\star}$ & 20.7 & 20.7 & 15.2 & 15.3 & $0.007^{\star}$ \\
\hline Hypolipidemic medication (\%) & 6.8 & 4.6 & 8.9 & 6.2 & $0.125^{\star}$ & 3.7 & 1.7 & 1.7 & 2 & $0.041^{*}$ \\
\hline Type 2 diabetes (\%)† & 4.0 & 0 & 4.2 & 1.8 & $<0.001^{*}$ & 4.2 & 3.5 & 3.0 & 1.8 & $<0.001^{*}$ \\
\hline SCORE risk score (\%) & $2.6 \pm 1.0$ & $1.3 \pm 0.4$ & $1.4 \pm 0.5$ & $1.3 \pm 0.5$ & $<0.001^{\star}$ & $0.5 \pm 0.2$ & $0.2 \pm 0.1$ & $0.2 \pm 0.1$ & $0.2 \pm 0.1$ & $<0.001^{*}$ \\
\hline Framingham risk score (\%) & $14.7 \pm 6.0$ & $7.4 \pm 2.3$ & $8.1 \pm 3.2$ & $7.5 \pm 3.4$ & $<0.001^{*}$ & $5.9 \pm 3.9$ & $3.0 \pm 2.1$ & $3.2 \pm 1.9$ & $3.3 \pm 1.9$ & $<0.001^{\star}$ \\
\hline \multicolumn{11}{|c|}{$\begin{array}{l}\text { The values are presented as mean } \pm \text { SD unless otherwise indicated. } \\
\text { *BMI is adjusted for when testing for differences between groups. } \\
\text { tIncluded in Framingham risk score. } \\
\text { †Included in SCORE risk score. } \\
\text { §Use of antihypertensive and lipid profile-lowering medication is adjusted for when testing for differences between groups. } \\
\text { BMI, body mass index; BP, blood pressure; CVD, cardiovascular disease; HDL, high-density lipoprotein; LDL, low-density lipoprotein; SCORE, Systematic Coronary Risk Evaluation; WHR, waist } \\
\text { to hip ratio. }\end{array}$} \\
\hline
\end{tabular}


Figure 2 Absolute risk of a first cardiovascular event in the next decade as defined by the Framingham risk score. BMI was adjusted for when testing for differences between groups with different smoking histories.

${ }^{\star} \mathrm{p}<0.001$. BMI, body mass index.

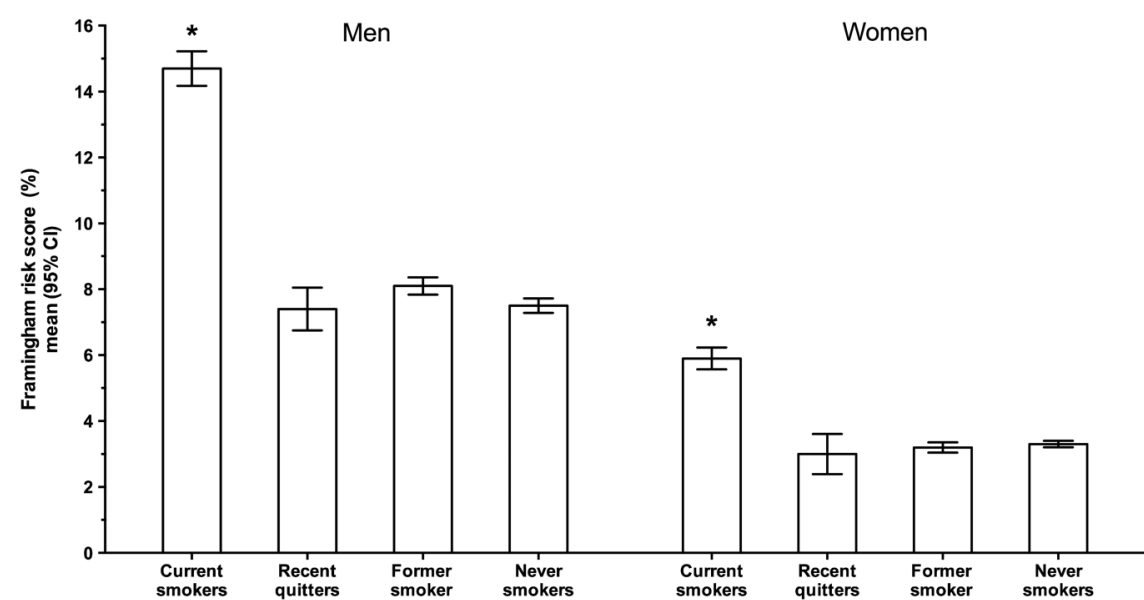

women) continued smoking at age 46 . Over $80 \%$ of those who had quit smoking before age 31 remained non-smokers at age 46 (84\% of men; $84 \%$ of women; figure 3).

\section{Changes in risk factors over time}

All CVD risk factors tracked between ages 31 and 46 (blood pressure, total cholesterol, LDL and HDL values, triglycerides, BMI, hip and waist circumference) increased with age. The greatest numerical difference was observed among current smokers, but there were no statistical differences between the groups.

\section{DISCUSSION}

In the present study, we set out to investigate CVD risk factors among men and women aged 46 years based on their smoking history.

\section{Smoking history and cardiovascular risk factors}

Smoking, lipid abnormalities and high blood pressure are well-established risk factors for CVD. Apart from being an individual risk factor for CVD, smoking has generally been associated with many of the other risk factors such as a poorer lipid profile and elevated blood pressure. According to previous research, smokers have higher serum cholesterol, triglyceride and LDL levels, and lower levels of HDLs than non-smokers, but the effect of smoking cessation on lipid profile seems to be quite modest. $^{12-14}$ A meta-analysis of 27 prospective studies on the effect of smoking cessation found a small but significant increase in the level of HDL, favouring former smokers over current smokers when the measurement intervals ranged from 30 days to 2 or more years. ${ }^{13}$ The same study found no significant changes in the level of total cholesterol, LDL or triglycerides. In the present analysis, we observed statistically significant differences in lipid levels, favouring individuals who had never smoked over current smokers. However, these differences were modest and clinically insignificant at the group level. Interestingly, lipid levels were slightly above recommendations in all groups studied. ${ }^{15}$ The use of antihypertensive or hypolipidemic medication was slightly more common among current smokers when compared with the group of never smokers. Also, type 2 diabetes was more common among smokers than never smokers, contributing to the higher Framingham total score in this group.

In the present study, there were no clinically major differences in blood pressure between the groups, and the mean blood pressure was within recommendations in all groups. ${ }^{15}$ Smoking causes acute increase in blood pressure, which declines quickly after smoking. ${ }^{16}$ However, there is no consensus regarding the role of smoking in long-term blood pressure in generally healthy people. ${ }^{17}$ Though lifelong non-smokers seem to have lower blood pressure than current smokers, the effect of smoking cessation on blood pressure remains unclear with contradictory findings. ${ }^{13-19}$ In one study, no significant change in blood pressure was found after two and a half years after smoking cessation in either the group of former smokers or the control group of never smokers. ${ }^{13}$ We were not able to find such a long-term effect in our population aged 46 years either.

Obesity and diabetes are also known to contribute to the absolute risk of CVD. ${ }^{12}$ In the present study, the group of former smokers had the highest BMI. Indeed, people who stop smoking often gain weight, which is suppressant. $^{20}$

While the risk of CVD decreases immediately after smoking cessation, the extent and speed of the risk reduction are not clear. ${ }^{21}$ Depending on the study, the CVD risk of former smokers reverts to the level of lifelong non-smokers within 3-15 years. ${ }^{21}{ }^{22}$ In our study, the total increase in CVD risk among current smokers resulted mainly from the smoking itself, rather than from the aggregation of multiple risk factors in these individuals. Therefore, our results support the assumption that smoking is predominantly a major individual risk factor for CVD, instead of increasing other risk factors or being an indicator for harmful lifestyle habits. $^{2}$ Indeed, contrary to our hypothesis, the differences in individual risk factors for CVD were not probably due to nicotine acting as an appetite 


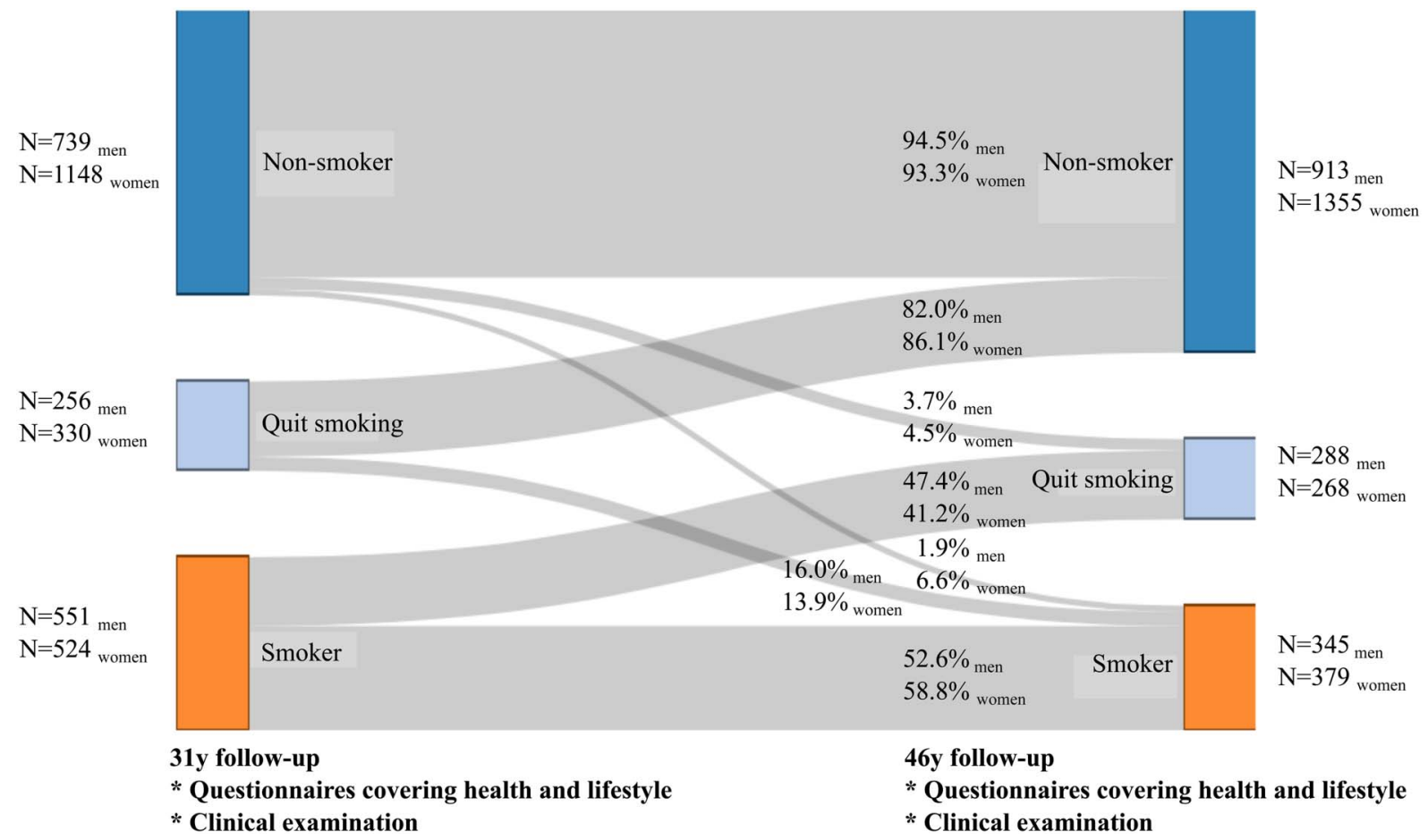

Figure 3 Changes in smoking behaviour over time. Categories for this figure were generated as follows: (A) At the age of 31, the 'non-smoker' group includes all individuals who had never smoked. At the age of 46 , the 'non-smoker' group includes individuals who had never smoked or who had quit smoking before the age of 31. (B) At the age of 31, the 'quit smoking' group includes participants who were 'recent quitters' or 'former smokers' at the time of evaluation. At the age of 46, the 'quit smoking' group includes those individuals who had smoked regularly at some point but had quit smoking between ages 31 and 46 . (C) The 'smoker' group includes individuals identified as 'current smokers' at the time of evaluation.

clinically significant among smokers, non-smokers, former smokers and recent quitters.

\section{Mechanisms of smoking-induced cardiovascular events}

The mechanisms underlying cigarette smoke-induced atherosclerosis, arterial thrombosis and their clinical manifestations as CVD are only partially understood and can be divided into several systemic pathways. First, reactive oxygen species induce cardiac remodelling, seen as left ventricular hypertrophy and atrial fibrosis, which increase the risk of stroke. ${ }^{23}$ Oxidative stress caused by systemic oxygen free radicals causes systemic and local inflammation, which is seen as elevation in levels of $\mathrm{C}$ reactive protein and peripheral leucocytes, in addition to other proinflammatory cytokines, which promote arterial thrombosis. ${ }^{24}$ Second, nicotine-stimulated release of adrenal medulla hormones modifies cardiac output by increasing heart rate, ventricular contractility and blood pressure, which might lead to cardiac ischaemia. ${ }^{25}$ Third, compounds in cigarette smoke expose smokers to myocardial ischaemia by causing deficient vasomotor functions, which is seen as thickening of the arterial wall and intima media, reduced ability to expand and contract vessels, as well as increased arterial stenosis. ${ }^{26} 27$ There are currently also many other known mechanisms by which smoking contributes to the increased CVD risk. ${ }^{28} 29$

\section{Strengths and limitations of the study}

While the rate of cardiovascular mortality is at a steady decline in the Western world, CVD remains the leading cause of death in our Finnish setting. ${ }^{30}$ Even though cardiovascular events lead to death all the more seldom, they are extremely costly for the healthcare system. Moreover, especially non-fatal stroke can have devastating consequences for the quality of life of the patient and their family. It is thus of key importance to recognise which measures are impactful in CVD prevention.

Large, prospective, population-based study samples, such as the Northern Finland Birth Cohort used in the present study, are optimal for studying common diseases and their risk factors. In the present sample, the participants' smoking history and changes to it were thoroughly mapped throughout their lives. Considering the fact that participation in the study takes several days at each point of data collection, the dropout rate has been very small. This may indicate high motivation of the participants, which is likely to increase the reliability of the self-reported data on smoking status and history. On the other hand, it is also possible that individuals with little interest in health issues may be under-represented in the sample. In the present study, we deliberately did not adjust analyses for the participants' socioeconomic status, which was expected to further highlight the differences in CVD risk factors between the groups. This is 
because smoking and other harmful health behaviour, such as an unhealthy diet, high alcohol consumption or lack of physical exercise, typically occur in lower socioeconomic classes. ${ }^{6}$ However, we did adjust for a common indicator of these harmful habits-BMI-when comparing individual risk factors and absolute risk scores between people with different smoking histories. Adjusting for family history might have further strengthened our results but could not be done with our data.

\section{Clinical conclusions}

Our aim was to characterise gender-specific CVD risk factors in relation to smoking behaviour and history in a large population-based Finnish study sample. Many risk factors were elevated among smokers in comparison with never smokers or to the group of participants who had quit smoking. However, these differences were modest and are not likely to have major clinical implications. The difference in these risk factors was so small between those who had smoked in some point in their lives but later quit and those who had never smoked that a statistically significant difference in absolute CVD risk for these groups could not be detected. Many deleterious changes caused by smoking are relatively quickly reversible, and therefore smoking cessation could have a great significance in reducing cardiovascular morbidity and mortality. In the UK, a $35 \%$ decline in smoking prevalence over two decades has resulted in nearly 30000 (24\%) fewer coronary heart disease deaths. This effect was much greater than that achieved by lowering cholesterol, which resulted in some 4700 (4\%) fewer deaths. ${ }^{31}$ Based on the results presented here, only modest aggregation of other CVD risk factors exists in smokers aged 46 years when compared with never smokers or ex-smokers. Therefore, quitting smoking at this age might even decrease CVD risk to approximately the same level with non-smokers.

Acknowledgements This study was sponsored by Pfizer Oy Finland. Northern Finland Birth Cohort 1966 received financial support from the Academy of Finland, University Hospital Oulu, University of Oulu, Finland, ERDF European Regional Development Fund-Well-being and health: Research in the Northern Finland Birth Cohort 1966 (grant no. 539/2010 A31592, 01.01.2011-31.12.2013). JK is an employee of a medical agency that provides consultation to several pharmaceutical companies, including Pfizer Oy Finland. HV is an employee of a medical agency that provides consultation to several pharmaceutical companies, including Pfizer Oy Finland. TY-o is the CEO of a medical agency (MedEngine Oy). TY-o has also worked as a consultant for Pfizer Oy Finland, Oy Bristol-Myers Squibb (Finland) Ab, Otsuka Pharmaceutical Scandinavia AB, Takeda Oy, and Teva Finland Oy. Markku Timonen has been reimbursed by Oy Bristol-Myers Squibb (Finland) Ab, H. Lundbeck A/S, Pfizer Oy Finland and Servier Finland Oy for attending four conferences; has been reimbursed by Astra Zeneca, Oy Bristol-Myers Squibb (Finland) Ab, Eli Lilly, Pfizer Oy and Servier Finland Oy for speaking on different educational occasions; has received advisory board fees from H. Lundbeck A/S and Pfizer Oy Finland for four meetings; and is a minor shareholder in Valkee Ltd. KL is an employee of Pfizer Oy Finland.

Contributors JK, HV, TY, SK-K and MT conceived the original idea for the paper. JJ and JA conducted the data analyses. JK and HV produced the initial draft of the paper. All authors were involved in the interpretation of the analyses and writing of the paper. All authors approved the final version of the paper and take responsibility for its content.
Funding University Hospital Oulu, Pfizer Oy Finland, Suomen Akatemia, Oulun Yliopisto, ERDF European Regional Development Fund (grant no. 539/ 2010 A31592).

Competing interests At the time of the study, JK was an employee of a medical agency that serves several pharmaceutical companies. Please find full list of competing interests of all authors in the manuscript.

Ethics approval Ethical Committee of the Northern Ostrobothnia Health District.

Provenance and peer review Not commissioned; externally peer reviewed.

Open Access This is an Open Access article distributed in accordance with the Creative Commons Attribution Non Commercial (CC BY-NC 4.0) license, which permits others to distribute, remix, adapt, build upon this work noncommercially, and license their derivative works on different terms, provided the original work is properly cited and the use is non-commercial. See: http:// creativecommons.org/licenses/by-nc/4.0/

\section{REFERENCES}

1. Mathers CD, Loncar D. Projections of global mortality and burden of disease from 2002 to 2030. PLoS Med 2006;3:e442.

2. Wong ND. Epidemiological studies of $\mathrm{CHD}$ and the evolution of preventive cardiology. Nat Rev Cardiol 2014;11:276-89.

3. Ezzati M, Henley SJ, Thun MJ, et al. Role of smoking in global and regional cardiovascular mortality. Circulation 2005;112:489-97.

4. WHO Report on the Global Tobacco Epidemic. 2013 (accessed 31 Dec 2014). http://www.who.int/tobacco/global_report/2013/en/

5. Lerman C, Niaura R. Applying genetic approaches to the treatment of nicotine dependence. Oncogene 2002;21:7412-20.

6. Jha P, Peto R, Zatonski W, et al. Social inequalities in male mortality, and in male mortality from smoking: indirect estimation from national death rates in England and Wales, Poland, and North America. Lancet 2006;368:367-70.

7. Jousilahti P, Vartiainen E, Tuomilehto J, et al. Sex, age, cardiovascular risk factors and coronary heart disease: a prospective follow-up study of 14786 middle-aged men and women in Finland. Circulation 1999;99:1165-72.

8. Health statistics for the Nordic countries 2013. Nordic Medico-Statistical Committee 100:2013 (accessed 2 Dec 2015). http://www.nowbase.org/ /media/Projekt\%20sites/Nowbase/ Publikationer/Helse/Health\%20Statistics\%202013.ashx

9. Lahti-Koski M, Pietinen P, Heliövaara M, et al. Associations of body mass index and obesity with physical activity, food choices, alcohol intake, and smoking in the 1982-1997 FINRISK Studies. Am J Clin Nutr 2002;75:809-17.

10. D'Agostino RB, Vasan RS, Pencina MJ, et al. General cardiovascular risk profile for use in primary care: the Framingham Heart Study. Circulation 2008;117:743-53.

11. Conroy RM, Pyörälä K, Fitzgerald AP, et al., SCORE project group. Estimation of ten-year of fatal cardiovascular disease in Europe: the SCORE project. Eur Heart J 2003;24:987-1003.

12. Jousilahti $\mathrm{P}$, Tuomilehto $\mathrm{J}$, Vartiainen $\mathrm{E}$, et al. Body weight, cardiovascular risk factors and coronary mortality: 15-year follow-up of middle-aged men and women in eastern Finland. Circulation 1996;93:1372-9.

13. Green MS, Harari G. A prospective study of effects of changes in smoking habits on blood counts, serum lipids and lipoproteins, body weight and blood pressure in occupationally active men. The Israeli CORDIS study. J Clin Epidemiol 1995;48:1159-66.

14. Maeda K, Noguchi Y, Fukui T. The effects of cessation from cigarette smoking on the lipid and lipoprotein profiles: a meta-analysis. Prev Med 2003;37:283-90.

15. Dyslipidemia (online). Current Care Guidelines. Working group set up by the Finnish Medical Society Duodecim and the Finnish Cardiac Society. Helsinki: The Finnish Medical Society Duodecim, 2014 (accessed 02/2015). http://www.kaypahoito.fi

16. Kool MJ, Hoeks AP, Struijker Boudier HA, et al. Short- and long-term effects of smoking on arterial wall properties in habitual smokers. J Am Coll Cardiol 1993;22:1881-6.

17. Lee DH, Ha MH, Kim JR, et al. Effects of smoking cessation on changes in blood pressure and incidence of hypertension: a 4-year follow-up study. Hypertension 2001;37:194-8.

18. Omvik P. How smoking affects blood pressure. Blood Press 1996;5:71-7.

19. Primatesta P, Falasschetti E, Gupta S, et al. Associations between smoking and blood pressure. Evidence from the health survey for England. Hypertension 2001;37:187-93. 
20. Mineur YS, Abizaid A, Rao Y, et al. Nicotine decreases food intake through activation of POMC neurons. Science 2011;332:1330-2.

21. Mannan $\mathrm{H}$, Stevenson C, Peeters A, et al. Framingham risk prediction equations for incidence of cardiovascular disease using detailed measures for smoking. Heart Int 2010;5:e11.

22. Honjo $\mathrm{K}$, Iso $\mathrm{H}$, Tsugane $\mathrm{S}$, et al. The effects of smoking and smoking cessation on mortality from cardiovascular disease among Japanese: pooled analysis of three large-scale cohort studies in Japan. Tob Control 2010;19:50-7.

23. Varela-Carver A, Parker $\mathrm{H}$, Kleinert $\mathrm{C}$, et al. Adverse effect of cigarette smoke and induction of oxidative stress in cardiomyocytes and vascular endothelium. Curr Pharm Des 2010;16:2551-8.

24. Libby P, Ridker PM, Maseri A. Inflammation and atherosclerosis. Circulation 2002;105:1135-43.

25. Benowitz NL. Cigarette smoking and cardiovascular disease: pathophysiology and implications for treatment. Prog Cardiovasc Dis 2003;46:91-111.
26. Pittilo RM. Cigarette smoking, endothelial injury and cardiovascular disease. Int J exp Pathol 2000;81:219-30.

27. Rahman MM, Laher I. Structural and functional alteration of blood vessels caused by cigarette smoking: an overview of molecular mechanisms. Curr Vasc Paharmacol 2007:5:276-92.

28. Csordas A, Bernhard D. The biology behind the atherothrombotic effects of cigarette smoke. Nat Rev Cardiol 2013;10:219-30.

29. Messner B, Bernhard D. Arteriosclerosis, thrombosis and vascular biology. J Am Heart Association 2014;34:509-15.

30. Official Statistics of Finland (OSF). Causes of death [e-publication] ISSN=1799-5078. 2014. Helsinki: Statistics Finland (accessed 6 May 2016). http://www.stat.fi/til/ksyyt/2014/ksyyt_2014_2015-12-30_ tie 001_en.html

31. Unal B, Critchley JA, Capewell S. Modelling the decline in coronary heart disease deaths in England and Wales, 1981-2000: comparing contributions from primary prevention and secondary prevention. BMJ 2005;331:614-20. 